\title{
REAL LINK OF A HIGH DATA RATE OFDM MODEM: DESCRIPTION AND PERFORMANCE
}

\author{
I. Pérez-Álvarez ${ }^{\dagger}$, J. López-Pérez ${ }^{\dagger}$, S. Zazo*, I. Raos*, B. Pérez-Díaz ${ }^{\dagger}$, E. Jiménez-Yguacel ${ }^{\dagger}$ \\ ${ }^{\dagger}$ Universidad de Las Palmas de Gran Canaria(ULPGC). CeTIC. Dpto. Señales y Comunicaciones. Las Palmas (Canary \\ Islands). Spain. Tel: +34-928457362, email: iperez@cetic.eu \\ * Universidad Politécnica de Madrid (UPM). Dpto. SSR. ETSI de Telecomunicación. Madrid. Spain. Tel.: +34-915495700 \\ Ext 4042, email: santiago@gaps.ssr.upm.es
}

Keywords: OFDM, probe modem, data modem, Automatic Link Management (ALMA).

\begin{abstract}
This paper deals with the design of an integrated system that is able to transmit data with high data rate and also high quality. However, the main feature is not the enhanced performance but the objective of providing a nearly permanent and reliable communication over time. In order to overcome the long term fading, a probe modem is simultaneously monitoring all the available channels and ranking them in terms of feasibility. A general manager named ALMA (Automatic Link MAnagement) detects when the current transmission is being degraded and in a transparent way to the user, decides when and how the channel change happens. To make this approach feasible, we have developed our own broadband transceiver that is able to transmit / receive simultaneous streams. In this paper we will describe the performance of the probe modem, the criteria and strategy for channel changing and the performance of the stable data modem.
\end{abstract}

\section{Introduction}

Communication in High Frequency (HF) band is not considered to be reliable, due to channel's great variations and highly dispersive characteristics, frequency selectivity, Doppler spread and multipath propagation. Briefly, it can be stated that all inconveniences of wireless channel are present, making this channel a very hostile environment.

Digital signal processing tools made possible more reliable transmission over wireless channels. Multicarrier modulation, specifically Orthogonal Frequency Division Multiplex (OFDM), is an appropriate approach for combating those wireless channel's characteristics.

Wireless channels are usually frequency selective, meaning that channel affects signals at different frequencies in a different manner. This requires complex equalisers for single carrier modulations. Multicarrier modulation schemes were proposed a long time ago to combat this obstacle, but its implementation was made possible just recently with digital signal processing methods. The idea is quite simple, yet very effective to avoid complex receivers and to provide more reliable communication. By sending in parallel manner a set of narrowband signals with bandwidth narrow enough, channel frequency characteristic can be approximated as flat in each signal bandwidth, and each signal will experience flat fading. This modulation leads to simple channel equalisation in frequency domain, as it consists of one complex coefficient per subcarrier that compensates channel's influence. OFDM is single user multicarrier modulation based on specific frequency separation of carriers modulated with different signals. If this distance equals the inverse of the symbol duration, orthogonality between different signals is achieved.

Although frequency selective channel are well coped with OFDM, the HF channel is even worse because it suffers from flat fading that attenuates completely the whole band. The only way to face this aspect is exploiting diversity, that enables to transmit over, desirably uncorrelated, channels. Instead of doing it blindly, our proposal monitors all the available set of channels and decides the changing procedure accordingly.

This paper describes the design principles and performance over a real link of an adaptive data rate modem that is able to guarantee stable communication with very high data rate via channel selection capability. The main feature of the system is the integrated functionality of data transmission over a certain selected channel and the periodical monitoring of the rest of the available channels. To make this approach feasible we have developed our own broadband transceiver (see [1] in this conference) that allows us to transmit and receive simultaneous transmissions. The obvious approach of this functionality is to transmit the same data via different channels to achieve frequency diversity or to multiplex uncorrelated transmissions over different channels to increase data rate. However, in this contribution we would like to address a different objective related to the design of permanent and reliable HF communications. For this purpose, one channel is devoted for data transmission while the rest of available channels are also periodically monitored using a similar waveform as a frequency hopping sounder. This way, the system manager (labelled ALMA Automatic Link Manager resembling the ALE concept) is able to select dynamically the most suitable channels depending on the individual SNR, multipath and Doppler rate.

Once the channel is selected a very robust modem has been developed using OFDM modulation. Most of our experience comes from the design of MC-CDMA $\mathrm{HF}$ modems intending to provide satisfactory performance for delay sensitive transmission as digital voice transmission (see [2-3]). Under that constraint, the multiuser detector achieves 
all the available diversity without resorting to long interleavers neither coding. However, for data transmission where delay is not so critical, we have recalled the OFDM modulation with adjustable modulation (4QAM, 16QAM, 64QAM) depending on the actual channel monitoring or the demanded data rate and very powerful LDPC codes. We have tested that LDPC incorporating log-likelihood ratios (LLR) provides much better performance than standard convolutional codes with not so long interleaver. This performance has been evaluated over real links between Madrid and Las Palmas de Gran Canaria (separated 1800 $\mathrm{Km}$ ) providing several statistics about the link quality depending on time parameters for all the assigned frequencies by the authorities for our research.

\section{General Scheme}

This section presents the global description of the system as can be observed in Figure 1.

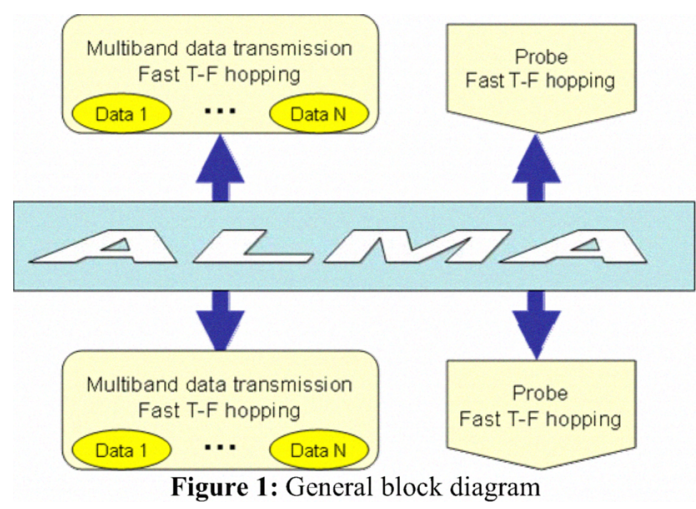

As we have already mentioned in the introduction section the system works as follows:

When a communications is started, the probe modem hops from one channel to the other evaluating the degree of quality and suitability for data transmission. The criteria for the ranking process is based on three main parameters: the SNR, the multipath and the Doppler. The estimate of these parameters is presented in a companying paper [4] but basically it is able to discriminate all the significant paths using a non linear least squares procedure and after that, Doppler profile per path is also determined. Basically we test that Doppler speed is slow enough in order to enable the designed pilot density and channel interpolating strategy to work properly and also, that delay spread is within the cyclic prefix of the OFDM symbol. For all those channels that fulfil these requirements, the one with maximum SNR is selected. The rest of the channels are properly ranked using all this information. You can observe that it is basically the functionality of an ALE but where the selection of the best channel takes into account the specific features of the used waveform.

Once, the channel is selected, depending on the required data rate and according to the channels state, the system initiates data transmission over one or more channels. In this document we are going to report just transmission over one channel but for instance in [5] we report multiband transmission for RADAR data application. Performance and design criteria of the modem will be presented in the sequel.

In a standard ALE system, once the channel is selected there is no further call to the ALE just in case the communication fades completely and it is necessary to re-start the process. Our intention is to control this situation and avoid the complete communication fading and make the user to feel that there is a permanent and stable link. For that purpose, the probe modem is all the time hopping over all the channels, evaluating their characteristics in a similar way as described during the acquisition period. This way we have a continuously updated table that ranks all the available channels. The probe signal length must be determined according to the number of channel to be monitored and the average Doppler profile.

The data modem is also evaluating the characteristic of the current channel using the channel estimate retrieved from the pilots and the SNR estimate obtained from the decided symbols (in terms of the Euclidean distance from the transmitted constellation). When it observes that quality degrades, it sets up a flag indicating the ALMA that a channel interchange must be coordinated. The ACKs should incorporate the information required to manage the new situation and coordinate when and to which channel the communication should be changed.

\section{Probe modem. Design}

This modem is basically a plain OFDM modem following the same structure of our voice modem. That modem is described in detail in $[2,3]$ and uses a MC-CDMA signal that it is shown to be very powerful in delay sensitive communications. Behind the MC-CDMA there is an OFDM structure with 73 carriers (60 data and 13 interleaved pilots), frequency separation $37.5 \mathrm{~Hz}$ and more than 3 miliseconds cyclic prefix length. The synchronization procedure in time and frequency and the channel estimation and interpolation process have probed to be very robust under real links and therefore are maintained identical.

The frame is formed by a header that is identical to the one described for the voice application including a single carrier interval for coarse offset estimation and two OFDM symbols for fine time and frequency estimation, and two more symbol for channel estimation. After that, $M$ symbols could be added to transmit side and control information between the two ends. After that a set of known symbols are used for monitoring in order to provide the means to estimate the frequency response and accordingly the delay and Doppler profile. The frame ends with an 'end of frame' symbol to be detected as the end of the transmission.

\section{Data modem. Design}

This modem basically follows also an OFDM structure with similar parameters in terms of synchronization and channel estimation as the voice and probe modems. However, there are important enhancements that must be described in order to deal with data transmission instead of voice signals. Clearly, error rates are more critical (in voice signals using a MELP a 
BER around to $10^{-2}$ is enough in order to asses the quality of the communication. Also, data is typically not limited by severe delay constraints. Both issues focused our attention to the application of a robust coding strategy. On the other hand, in order to provide a wide range of performances, other constellations should be implemented (the voice application just considered 4QAM).

Regarding the code, we compared the performance of a convolutional code $(133,171)$ (used also for WiMAX and IEEE $802.11 \mathrm{a} / \mathrm{b} / \mathrm{g}$ ) with code rate $1 / 2,4 / 5$ (with puncturing) and also a LDPC with equivalent code rates. Both cases use LLR as soft bits to improve performance. Clearly, LDPC outperforms the CC for all the constellations, delays (we considered 3 cases: E1 1.6 msec., E2 3.3 msec. and E3 5 msec. with random block interleaving) and CCIR channels (good, moderate and bad). Due to the lack of space we just present as an example the bad channel for both code rates:

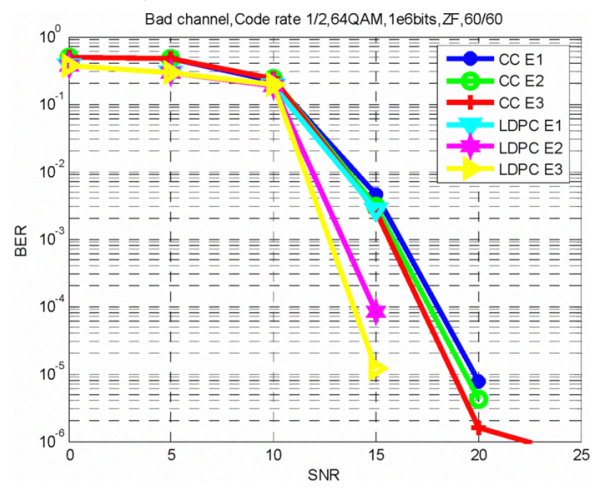

Figure 2. Bad channel. Code rate 1/2

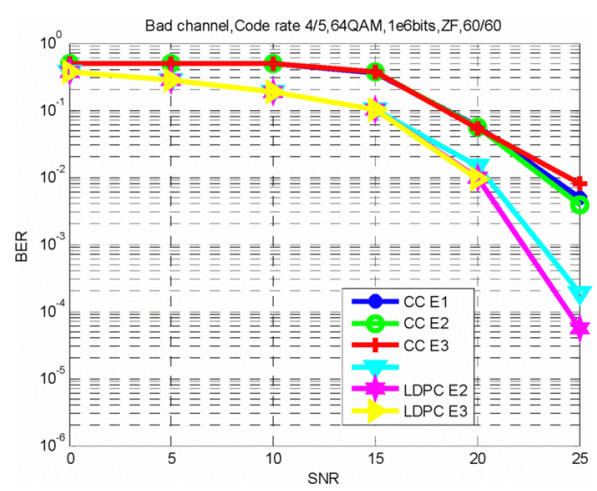

Figure 3. Bad channel. Code rate $4 / 5$

Data rates achievable are shown in the following table:

\begin{tabular}{|c|c|c|c|c|}
\hline \multirow{2}{*}{ Coding } & \multirow{2}{*}{$\begin{array}{l}\text { Code } \\
\text { Rate }\end{array}$} & \multicolumn{3}{|c|}{ Data Rate [b/sec] } \\
\hline & & 4QAM & 16QAM & 64QAM \\
\hline \multirow{2}{*}{ Conv. } & 0,5 & 1787 & 3574 & 5362 \\
\hline & 0,8 & 2867 & 5734 & 8602 \\
\hline \multirow{2}{*}{ LDPC } & 0,5 & 1800 & 3600 & 5400 \\
\hline & 0,8 & 2880 & 5760 & 8640 \\
\hline
\end{tabular}

Table 1: Data rates of the data modem
Next figure shows the block diagram of the system just to clarify the main aspects and procedures. Details regarding the calculation of the LLR are out of the scope of the paper. We just want to mention that we have implemented a variant of the standard 'Sum-Product' or 'Belief Propagation' named 'Min-Sum' where all the calculations take place in the logarithmic domain. It also uses the approximation of the maximum value of the logarithmic because, although suboptimum, it is much less complex and performance does not degrade significantly.

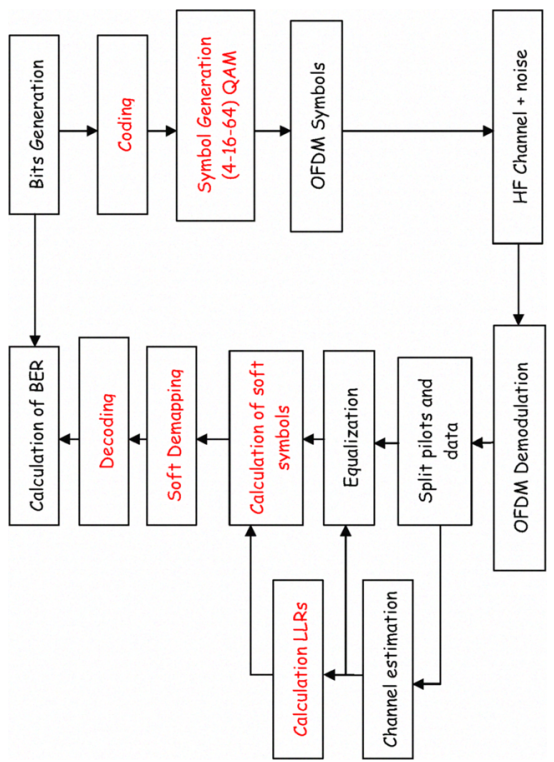

Figure 4. Block diagram of the data modem

\section{Probe modem. Real trials}

The campaign of real trials was very extensive and clearly we do not have the possibility to show all the results. Let us summarize some of the most representative achievements using the channel $18.201 \mathrm{KHz}$ (assigned to our groups) transmitting about 30 seconds length probe alternatively from Madrid to Las Palmas (transmission 1) and the other way round (transmission 2) along all the day.

Figure 5 shows the estimated SNR of transmission 2 (the other way round provide similar results).

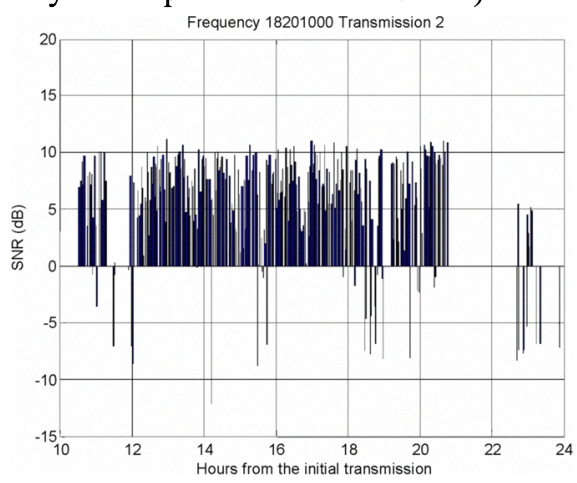

Figure 5. Measured SNR. LPA to MAD

Next figure will show the histograms of delay and Doppler for both links: 


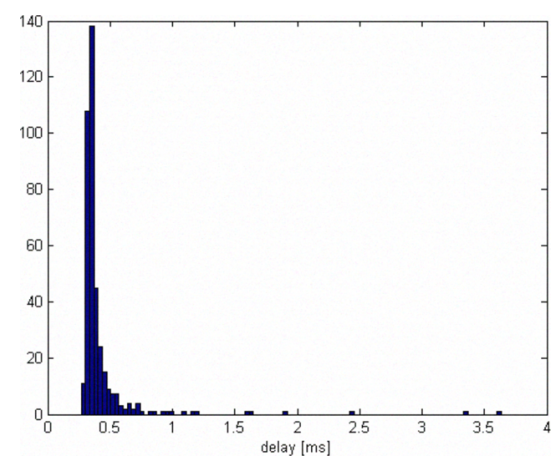

Figure 6. Delay histogram. LPA to MAD

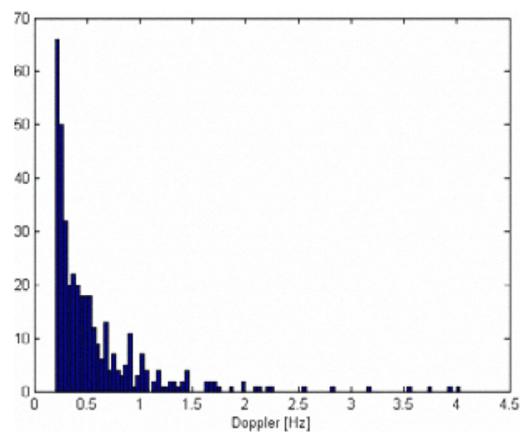

Figure 7. Doppler histogram LPA to MAD

In this case, we are going to present the same test in the other direction to remark the non reciprocity of the link. This issue has been discussed along the time and it can be concluded that it depends very much on the layer where the link takes place. According to our experience, this $1800 \mathrm{Km}$ link is non reciprocal. You can see [6] where some arguments in this sense are presented. We would like also to emphasize that in terms of SNR, the link is neither reciprocal. Depending on the location of our labs, Madrid (urban area) is much more noisy than Las Palmas (rural area).

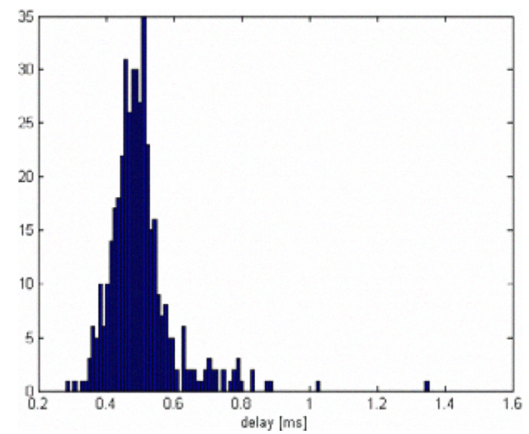

Figure 8. Delay histogram. MAD to LPA

We have also carried out a test to determine the probability of acquisition (receiver receives correctly the frame), hand-shaking (after acquisition when the receiver ACKs and the transmitter also receives correctly) and frame detection (the case where two simultaneous hand-shakes take place assuming the frame is composed of two, two directional, messages). Figure 10 shows the statistics of these tests:

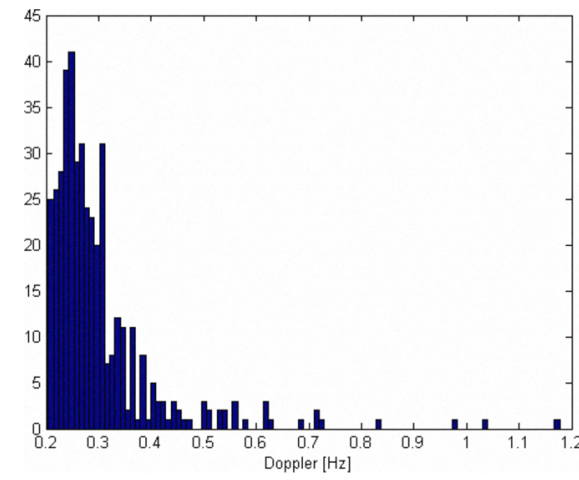

Figure 9. Doppler histogram. MAD to LPA

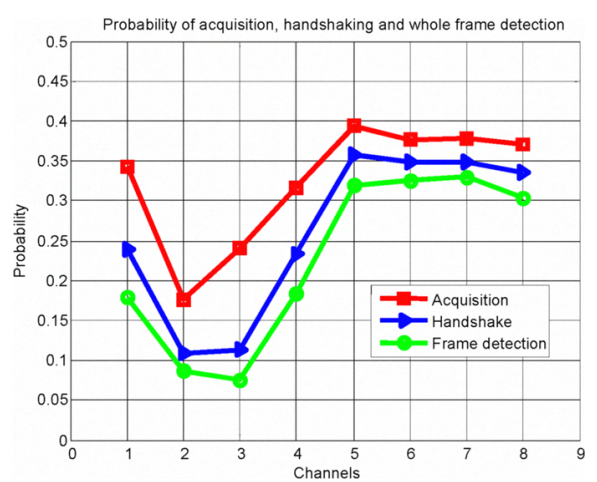

Figure 10. Statistics of connectivity

In figure 10 , Channel 1 to 4 represent transmission in the $10 \mathrm{MHz}$ band in different channels that are separated 3 $\mathrm{KHz}$, while channel 5 to 8 were carried out in the $18 \mathrm{MHz}$ band also separated $3 \mathrm{KHz}$.

Other test was developed in order to evaluate the probability of simultaneous transmission. The main problem that we suffered was the fact that we do not have assigned channels suitable for this test, so we have been transmitting over channel that were also used by other users playing the role of interferers. We have considered until four simultaneous channels separated $50 \mathrm{KHz}$ (although the broadband transceiver is able to cope with $1 \mathrm{MHz}$ bandwidth, we have to take into account the limited bandwidth of our antennas which is less than $300 \mathrm{KHz}$ ). Figure 11 shows the statistics along the day where we have set a threshold of $6 \mathrm{~dB}$ indicating that a link could be considered as satisfactory for SNR higher that that value. We have also to mention that these values, for instance $30 \%$ of two simultaneous transmissions is considering all the day long where the channel is blocked during the night. So, the correct analysis should conclude that when the channel is open ( $45 \%)$ there is about $2 / 3$ that we can make two-band transmission.

\section{Data modem. Real trials}

Real trials were carried out using channel 10.137 transmitting the same image (the well known Lena's picture) using different configurations. For instance we are going to show transmission using LDPC $1 / 2$ for 4-QAM, 16-QAM and 64QAM. Along with the visual effect it is shown the estimates of the channel where the transmission took place. 


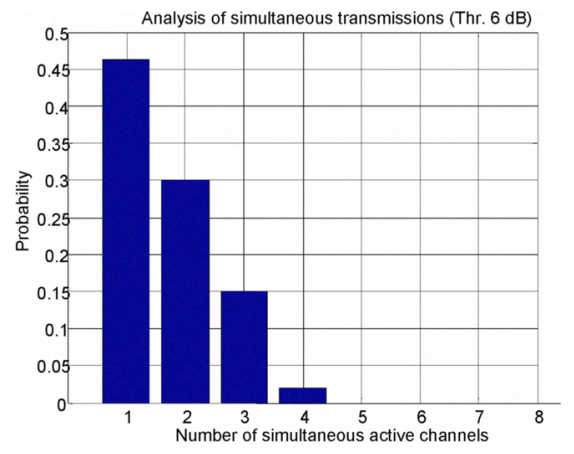

Figure 11. Statistics of simultaneous transmissions

Although all transmission took place sequentially, it can be observed that the channel for 64-QAM was much better than when using the 16-QAM, therefore the performance is surprisingly better. We will observe the transmitted image on the left side and the received one on the right side. The summary of the parameters and performances are presented in the following table

\begin{tabular}{|l|c|c|c|}
\hline Constellation & Av. Delay (ms) & Av. Doppler (Hz) & BER \\
\hline 4-QAM & 0.6 & 1.6 & $9.810^{-5}$ \\
\hline 16-QAM & 0.8 & 2 & $5.010^{-2}$ \\
\hline 64-QAM & 0.4 & 0.5 & $9.510^{-3}$ \\
\hline
\end{tabular}

Table 2. Performances of the data modem in three real trials

It can be observed that the channel when transmitting the 16-QAM was very fast and probably the channel interpolation process is not able to cope with these variations and performance degrades.
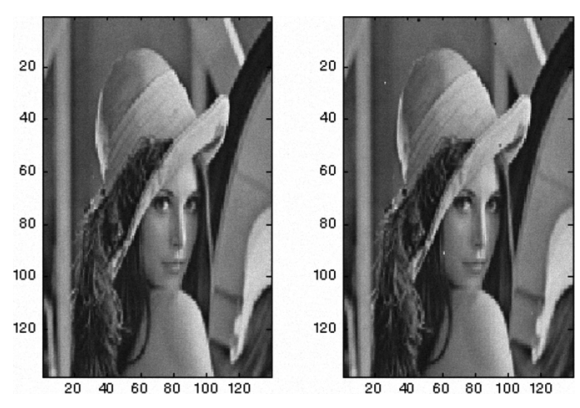

Figure: 12. LDPC 1/2. 4-QAM
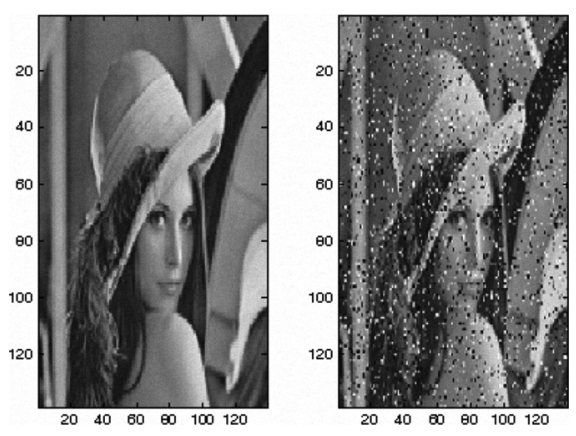

Figure: 13. LDPC 1/2. 16-QAM

\section{Conclusions}

This work presents an initiative to cope with one the most challenging topics in HF communications: provide a high data rate link stable and reliable. Increasing data rate has been accomplished by an enhanced OFDM modem with robust coding and soft decoding process via a LDPC. Reliability can be guaranteed by the simultaneous transmission of a probe over all the available channels that evaluate their suitability for transmission on line and a general manager (named ALMA) who is in charge of coordinating the swapping process. This performance has been tested in real links between Madrid and Las Palmas de Gran Canaria
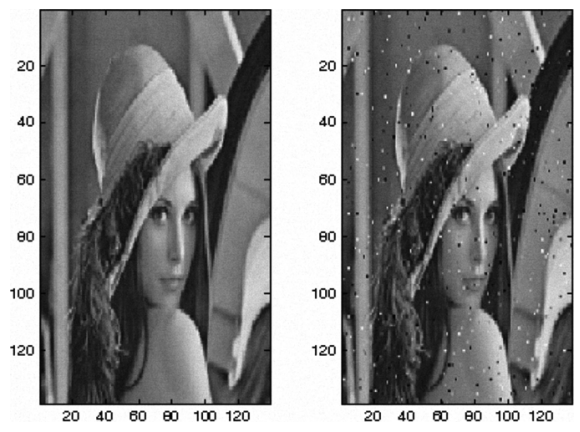

Figure: 14. LDPC $1 / 2.64-\mathrm{QAM}$

\section{Acknowledgements}

We would like to thank Aena for their support and also the Spanish Education and Science Ministry under Grant TEC2007-67520-C02-01/02/TCM

\section{References}

[1] Pérez-Díaz, B.; Jiménez-Yguacel, E.; López-Pérez, J.; Pérez-Álvarez, I.; Zazo-Bello, S.; Medieta-Otero, E. Design and construction of a broadband $(1 \mathrm{MHz})$ digital $\mathrm{HF}$ transceiver for multicarrier and multichannel modulations. IRST 2009: 11th International Conference on Ionospheric Radio Systems \& Techniques.Edinburgh, U.K.; June 2009.

[2] Santana, H.; Zazo, S.; Pérez, I.; Raos, I.; Mendieta, E.; López, J. Validation of a HF Spread Spectrum MultiCarrier Technology through real Link Measurements. European Trans. on Telecommunications (ETT), Vol17,Nov.-Dec. 2006 [3] Santana-Sosa, H.; Pérez-Álvarez, I.; A.; Mendieta-Otero, E.; López-Pérez, J.;Zazo, S.; Raos, I.; Performance over a real link of a HF software radio modem for interactive digital voice communications. HF 2006: Tenth International Conference on HF Radio Systems and Techniques 2006, London, UK, July 2006

[4] Sanz González, J.L..; Zazo-Bello, S.; Pérez-Álvarez, I.; López-Pérez, J.; Parameter estimation algorithms for ionospheric channels. IRST 2009: 11th International Conference on Ionospheric Radio Systems \& Techniques.Edinburgh, U.K.; June 2009.

[5] Zazo-Bello, S.; Raos, I. López-Pérez, J.; Pérez-Álvarez, I.; Aynchronous and variable data OFDM modem for RADAR data transmission. . IRST 2009: 11th International Conference on Ionospheric Radio Systems \& Techniques.Edinburgh, U.K.; June 2009.

[6] Jull, G. W. Non reciprocal characteristics of a $1500 \mathrm{Km}$ HF ionospheric path. Proceeding of the IEEE, March 1967. 